\title{
Segregación y Exclusión Escolar de la Primera Generación de Alumnado de Origen Migrante: De la Integración a la Desintegración
}

\author{
Segregation and School Exclusion of First Generation Pupils \\ with a Migrant Background: From Integration to Disintegration
}

\author{
Mohamed Chamseddine * \\ Universidad de Murcia, España
}

\begin{abstract}
Una revisión exhaustiva de las abundantes investigaciones y planes de formación sobre atención de la diversidad cultural, nos remite de forma persistente al concepto integración escolar del alumnado de origen migrante. No obstante, su trayectoria académica desigual y poco alentadora, nos conduce hacia una inexcusable desintegración y exclusión escolar de los mismos. La polarización y segregación escolar, el abandono del Sistema Educativo en Educación Secundaria y la baja tasa de dicho alumnado en la Universidad, confirman la desentonación temeraria producida en las últimas tres décadas, debido a la ausencia de calidad, equidad y eficacia educativa en la gestión de dicha materia. El objetivo de este trabajo es analizar de forma transversal los aspectos pedagógicos e institucionales, asociados a la atención al alumnado migrante, y su vínculo con la segregación y exclusión escolar de los mismos, como son la baja implicación de las familias migrantes en la dinámica escolar, la escasa formación docente, la rotación del profesorado, la ausencia de mediadores interculturales, etc. Todo ello, para impulsar la reflexión sobre posibles respuestas inclusivas, coherentes y contundentes, que garanticen el éxito escolar de la próxima generación. Por tanto, esta cuestión no sólo merece interés académico, sino que se trata de una urgencia moral, política y social.
\end{abstract}

Descriptores: Segregación; Alumnado migrante; Inclusión; Exclusión; Escuela.

\begin{abstract}
A comprehensive review of abundant researches and training plans about cultural diversity persistently refers us to students' integration school concept with a migrant background. However, their unequal and not very encouraging academic path lead us to an inexcusable disintegration and school exclusion of them. The polarization and segregation of schools, the dropout from the education system in secondary school and the low rate of such students at university confirm the reckless disharmony produced in the last three decades, due to the lack of quality, equity and educational efficiency in this subject management. The aim of this work is to analyse, in a transversal way, pedagogical and institutional aspects associated with the care of migrant students and their link with their segregation and exclusion from school, such as low involvement of migrant families in the school dynamic, scarce teacher training, teacher rotation, absence of intercultural mediators, etc. All of that is to encourage reflection on possible inclusive, coherent and forceful responses that will guarantee the school success of the next generation, but this question does not only deserve academic interest, also it is a matter of moral, political, and social urgency.
\end{abstract}

Keywords: Segregation; Migrant students; Inclusion; Exclusion; School.

*Contacto: mohamed.c.h@um.es

ISSN: $1696-4713$

www.rinace.net/reice/

revistas.uam.es/reice
Recibido: $\quad 20$ de abril 2020

$1^{\text {a }}$ Evaluación: 30 de junio 2020

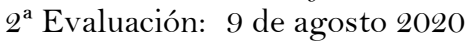

Aceptado: 25 de agosto 2020 


\section{Introducción}

La incorporación de España a los estados receptores de migrantes, se produjo en paralelo con el desarrollo económico y social que se inició en los años 90. Sin embargo, el impacto de los flujos migratorios no está distribuido de forma homogénea en las distintas Comunidades Autónomas, debido principalmente a las oportunidades de empleo disponibles en cada zona geográfica, especialmente en el sector agrario, el servicio doméstico y el sector del comercio. Dicha diferencia en la distribución se refleja del mismo modo, en los contextos escolares, cuyo alumnado extranjero procede de Latinoamérica, países del Magreb, África subsahariana, Asia Oriental, Europa del Este e incluso alumnos y alumnas procedentes de la propia Unión Europea (Consejo Económico y Social, 2019).

Dicho lo anterior, cabe indica que el eterno y controvertido debate sobre la calidad y la equidad educativa ha producido cambios y reformas en el sistema educativo, fruto de una sociedad dinámica y un escenario político cambiante. Desgraciadamente, este escenario no logra superar las desigualdades entre la inclusión de los privilegiados y la exclusión de los desfavorecidos que también aspiran a que le garanticen más y mejor educación. En una reflexión sobre la calidad educativa, Escudero (2014) sostiene que es un principio inherente al derecho a una educación digna, efectiva y democrática cuyo compromiso moral sería la satisfacción de las necesidades y expectativas de los discentes con consistencia y perfeccionamiento de ciertos procesos educativos, para la transformación y cohesión social. En cuanto a la equidad educativa, Sánchez-Santamaría y Manzanares (2012) manifiestan que se debería correlacionar positivamente la equidad de acceso, donde todos pueden acceder al sistema educativo; la equidad de oferta, que permite acceder a todos a las mismas oportunidades educativas, ajustando el nivel al ritmo y capacidades de aprendizaje de cada alumno y alumna; la equidad de proceso, que promueve y genera condiciones, experiencias y situaciones pedagógicas orientadas al éxito educativo para todos, garantizado la permanencia en el sistema educativo; y, por último, la equidad de resultados que garantice que todo el alumnado alcance las mismas metas a nivel de competencias básicas en las distintas etapas educativas.

No obstante, lograr el cumplimiento de estas aspiraciones de gran relieve y trascendencia social y educativa, implica garantizar la igualdad en los logros educativos y en las oportunidades, todavía a día de hoy, ausentes especialmente en el tema que nos ocupa. Así, la irrupción del alumnado migrante en los centros educativos españoles, es relativamente reciente en comparación con otros contextos europeos. Su incorporación cuenta ya con tres décadas de presencia en el sistema educativo, desde sus inicios en 1990 hasta 2020, constituyendo la primera generación de este "nuevo" alumnado. Ante la presencia de los mismos, el marco legal incorpora como novedades la no discriminación, y el respecto por la diversidad de procedencias, junto a la igualdad de oportunidades de género, como principios de la educación, en el artículo 2.3 de la Ley de Ordenación General del Sistema Educativo (LOGSE, 1990), estableciendo la efectiva igualdad de derechos entre los sexos, el rechazo a todo tipo de discriminación, y el respeto a todas las culturas. A continuación, se insiste en el derecho a la educación del alumnado migrante (4/2000) en su artículo 9.1 que pasamos a transcribir: los extranjeros menores de dieciséis años tienen el derecho y el deber a la educación, que incluye el acceso a una enseñanza básica, gratuita y obligatoria. Los extranjeros menores de dieciocho años también tienen derecho a la enseñanza postobligatoria. Y de forma persistente aparece la integración de los mismos en el artículo 
9.3, que establece que los poderes públicos promoverán que los extranjeros puedan recibir enseñanzas para su mejor integración social.

Ante la urgencia de dar respuesta a este fuerte incremento de alumnos extranjeros en las aulas en la primera década, el sistema educativo español ha ido poniendo en marcha de manera progresiva y a veces apresurada, distintas acciones y medidas en los centros educativos. Es lo que se afirma en el artículo 42.1 de La Ley de Calidad de la Educación (LOCE, 2002) que pasamos a transcribir: las Administraciones educativas favorecerán la incorporación al sistema educativo de los alumnos procedentes de países extranjeros, especialmente en edad de escolarización obligatoria. Para los alumnos que desconozcan la lengua y cultura española, o que presenten graves carencias en conocimientos básicos, las administraciones educativas desarrollarán programas específicos de aprendizaje, con la finalidad de facilitar su integración en el nivel correspondiente. Matizando en el artículo 42.2 que los programas a que hace referencia el apartado anterior se podrán impartir, de acuerdo con la planificación de las Administraciones educativas, en aulas específicas establecidas en centros que impartan enseñanzas en régimen ordinario. El desarrollo de estos programas será simultáneo a la escolarización de los alumnos en los grupos ordinarios, conforme al nivel y evolución de su aprendizaje.

Lejos de olvidarse de aquellos alumnos migrantes que presentan mayores riesgos de fracaso y abandono escolar estimulados por su incorporación tardía al sistema educativo, aparece la Ley Orgánica de Educación (LOE, 2006), en su artículo 78.1 en donde se afirma que corresponde a las administraciones publicas favorecer la incorporación al sistema educativo de los alumnos que, por proceder de otros países o por cualquier otro motivo, se incorporen de forma tardía al sistema educativo español. Dicha incorporación se garantizará, en todo caso, en la edad de escolarización obligatoria. Casi una década después, aparece el borrador de la Ley Orgánica 8/2013, de 9 de diciembre, para la mejora de la calidad educativa (LOMCE, 2013), que matiza en su artículo 2 la formación en el respeto y reconocimiento de la pluralidad lingüística y cultural de España y la interculturalidad como un elemento enriquecedor de la sociedad. Y recientemente, el Consejo de Ministros ha aprobado con fecha de 03/03/2020 un Proyecto de Ley Orgánica de Modificación de la Ley de Educación (LOMLOE, 2020) que pretende mejorar la calidad educativa siguiendo las directrices de la OCDE, y tal y como plantea la Agenda 2030, resaltando la necesidad de una distribución equilibrada del alumnado, así como reforzar la prevención de la segregación -algo que afecta a la población migrante- entre escuelas y entre las redes pública y concertada, para que todos los alumnos tengan las mismas oportunidades.

Repárese en que los distintos textos legales afirman su compromiso educativo y social sin exclusiones, algo que han recalcado los países miembros de la UNESCO en la Agenda Educativa 2030 "Asegurar una educación inclusiva y equitativa de calidad y promover oportunidades de aprendizaje a lo largo de la vida para todos y todas". En la práctica escolar, sin embargo, se confirman los tímidos avances, en donde pocos alumnos de origen migrante aspiran a cursar una titulación universitaria; los más resilientes confían en lograrlo y otros, apenas se lo plantean por las bajas expectativas en superar las dificultades en las distintas etapas del proceso formativo. Prueba de ello es la polarización y segregación escolar, el abandono escolar en Educación Secundaria y la baja tasa de dicho alumnado en la universidad. En tal sentido, se nos plantean los siguientes interrogantes interrelacionados entre sí, a los que trataremos de dar respuesta en el presente trabajo: ¿cuáles son los detonantes de la aparición de la polarización escolar?, ¿cuáles son los 
determinantes claves en el fracaso y abandono escolar de la población migrante?, ¿qué formación específica en atención al alumnado de origen extranjero recibe el profesorado?, ¿existe una comunicación fluida entre las familias migrantes y los docentes?, ¿qué tipo de medidas se aplican para gestionar la diversidad cultural en los centros educativos?; y, finalmente, ¿los centros educativos cuentan con mediadores interculturales para facilitar la inclusión educativa de dicho alumnado?.

Para profundizar en dichas cuestiones el presente artículo se vertebra en dos ejes transversales que indican las causas y consecuencias intrínsecamente asociadas a la exclusión escolar. El primero aborda los determinantes de la segregación y exclusión escolar de la población migrante, y el segundo trata los aspectos adicionales que actualmente dificultan la inclusión escolar de dicho alumnado.

\section{Las desigualdades en las tres etapas educativas}

Una de las desigualdades más citada en la bibliografía existente en la materia, tiene que ver con la aparición de la polarización escolar en determinados centros educativos de carácter público, fruto de la ambigüedad de normativas reales y adicionales de la política de escolarización de alumnado migrante extranjero (Chamseddine, 2018a; Salinas y Santín, 2012). Dicha tendencia creciente se alimenta a menudo de una serie de elementos como el éxodo masivo del alumnado autóctono a otros centros (Moreno, 2002), el criterio adicional de la libre elección de centro (Martín, 2010), la asociación de la segregación residencial con la polarización escolar (Ortiz, 2014), la inexistencia del reparto equitativo entre la red escolar pública y concertada (Ortiz, 2010); la violencia estructural de la política de zonificación (García et al., 2014), el pago obligatorio de cuotas mensuales (Franzé et al., 2012), unido a las medidas extras, como la reserva de plazas escolares, acuerdos y cooperación entre centros escolares para reducir la presencia de alumnado de origen extranjero en sus aulas (Rubia, 2013), lo que genera la creciente aparición de los centros llamados "línea uno".

En este sentido, la investigación desarrollada por Murillo, Martínez-Garrido y Belavi (2017) pone de manifiesto, que el sistema educativo español es obviamente inequitativo en términos de segregación entre comunidades autónomas. Estos autores revelan que Andalucía y Galicia encabezan la segregación escolar. A continuación, aparece una segregación significativa en la Comunidad Foral de Navarra, Cataluña, Principado de Asturias, Cantabria, Comunidad Valenciana, Castilla y León, Castilla-La Mancha, Islas Baleares, Comunidad de Madrid, Región de Murcia y Aragón. Y, por último, se encuentra la Rioja con menos niveles de segregación escolar.

Estas dificultades intencionadas (Essomba, 2014), vulneran reiteradamente el principio de igualdad, convirtiendo a los centros escolares en "casi-mercados educativos", como manifiesta Alegre (2010). En definitiva, se trata de toda una red de aspectos explicativos, entre otros, que definen las concentraciones y segregaciones escolares en numerosos centros educativos de titularidad pública, que conducen a menudo a la desintegración escolar y, por tanto, a la desintegración social de este "nuevo" alumnado. En este sentido, en el libro verde migración y movilidad: Desafíos (retos) y oportunidades para los sistemas educativos europeos (2008), se manifiesta que los centros educativos deberían garantizar una educación de calidad y equidad, desde una sociedad inclusiva que permita la 
integración, prosperidad y productividad de los alumnos extranjeros en su sociedad receptora. En tal sentido, Escudero (2006) insiste en:

\begin{abstract}
Asumir el compromiso de no mirar para otro lado allí donde se esté vulnerando el derecho básico a la educación; denunciar factores y dinámicas políticas, sociales y educativas de exclusión y discriminación en contextos globales y locales; denunciar la inmoralidad de los guetos sociales y escolares, urgiendo medidas de lucha contra la dualización escolar y educativa. (p. 31)
\end{abstract}

Como es bien sabido, la transición académica hacia educación secundaria produce un impacto muy significativo, especialmente en la población que se encuentra en una situación de mayor vulnerabilidad académica y social. En tal sentido, el alumnado de origen migrante se enfrenta a esta etapa de alto riesgo de abandono escolar, ya que forma parte de los candidatos al fracaso escolar, y más aún si son alumnos de incorporación tardía que se enfrentan a un sistema educativo desconocido. Las posibilidades del éxito escolar se reducen a la mitad, si el menor llega a España con menos de cinco años o bien si su llegada se produjo con más de 12 años. El fracaso y abandono escolar se multiplica a menudo por la ausencia de estrategias estimulantes y potenciadoras del desarrollar competencias resilientes en y desde los centros educativos de Educación Secundaria (Escudero, 2014).

En esta etapa educativa, los alumnos y alumnas de origen migrante corren el mismo riesgo que los autóctonos, con la diferencia que los primeros superan el $20 \%$-en la mayor parte de las Comunidades Autónomas- del alumnado que se marcha de las aulas sin conseguir el título de educación secundaria obligatoria. Es decir, al no alcanzar los objetivos de la E.S.O se incorporan a la desentonación temeraria y a las lagunas de las medidas pedagógicas inspiradas en el enfoque de compensación y diversificación educativa. Prueba de ello es el desfile en dicha etapa de los sucesivos programas que han arbitrado las distintas leyes educativas, como es el caso de los Programas de Diversificación Curricular (PDC), los Programas de Garantía Social (PGS), los Programas de Cualificación Profesional Inicial (PCPI), y, en la actualidad con la LOMCE, la Formación Profesional Básica (FPB) que en un principio se implantaría para minimizar el impacto del desfase del currículo regular de los alumnos y alumnas, pero han terminado convirtiéndose en muchos casos en el motor principal hacía el fracaso y abandono escolar. Para paliar dicha escena la OCDE insiste en su informe del Panorama de la Educación 2019, en que la educación es el mejor antídoto contra el desempleo. Por ello, el Ministerio de Educación cuadriplicó en el mismo año la inversión para minimizar el impacto del abandono escolar temprano donde supuestamente invirtió más de 81 millones de euros para afrontar la difícil tarea de mantener a los alumnos y alumnas escolarizados. Sin embargo, no deja de incrementarse la tasa de jóvenes inactivos, que no están integrados en los sistemas de formación, agudizando de esta manera su destino definitivo a empleos precarios, y por tanto, a su vulnerabilidad sociolaboral.

Asimismo, los chavales dejan de ser estudiantes, de la formación reglada, para controvertirse de manera "voluntaria" en usuarios- de la formación no reglada-, en los programas de Garantida Juvenil, conocidos como Programas Operativos de Empleo Juvenil (POEJ). Inicialmente, estos programas perseguían reducir el desempleo juvenil ofreciendo respuestas "formativas" y de inserción laboral. No obstante, estos jóvenes susceptibles de participar en esta iniciativa europea tampoco pueden acceder al itinerario formativos de los mismos, ya que el título de graduado en la ESO es un requisito imprescindible para incorporarse a la mayor parte de los talleres de formación. 
Dicho lo anterior, obviamente, la polarización y segregación escolar y el desenganche del sistema educativo en Educación Secundaria, ha producido la escasa visibilidad de dicho alumnado en la Educación Superior. En este sentido, el periódico La Verdad (2019) de la Región de Murcia, señala que la presencia de alumnado migrante en la Universidad empieza a tener más visibilidad en las distintas facultades; sin embargo, no supera el $8 \%$ de aspirantes a la Universidad en el caso de Murcia.

Así, hay que destacar que apenas existen estudios e investigaciones sobre alumnado de origen migrante en esta etapa educativa, ni datos desagregados en la propia página web de la Oficina de Estadística del Ministerio de Educación. En este sentido, aparece alumnado extranjero refiriéndose a aquel alumnado que viene a España con el objetivo único de realizar estudios en el estado español, sin haber cursado las dos etapas anteriores en el mismo. Autores como Cucalón y Del Olmo (2019) señalan que el profesorado afronta de manera sistemática y permanente dilemas y tensiones frente a alumnado de origen migrante, cuya expectativa es el acceso a la Universidad, teniendo en cuenta las serias limitaciones de la administración educativa para producir la igualdad de oportunidades. Del mismo modo, Machado (2015) considera que la diversidad cultural de este alumnado queda desprotegida por la ausencia de programas y medidas de apoyo, acogida, orientación y seguimiento en las Universidades Españolas. Sin embargo, a pesar de las desigualdades de acceso a las mismas, sus alumnos y alumnas de origen migrante se caracterizan por un nivel de estudio elevado, comparten y atraviesan determinadas experiencias similares, formando un grupo de privilegiados ante las adversidades entre la comunidad migrante (Vecina, 2005).

Otra limitación a destacar y apreciable en todas y cada una de las etapas educativas, se encuentra relacionada con la nacionalidad del alumnado y no con su procedencia. Es decir, que de nuevo el alumnado de origen migrante que posee la nacionalidad española, no queda reflejado en las estadísticas del Ministerio de Educación como alumnado migrante extranjero, sino como autóctono. Este determinante clave, agudiza la alarmante trayectoria académica desigual y poco alentadora, que viene a explicar la inexcusable desintegración y exclusión escolar de los mismos.

\section{Aproximación a otras variables implicadas en la exclusión escolar}

Es una evidencia insoslayable la numerosa literatura científica, en relación a la participación de las familias en la escuela a nivel general y de los progenitores migrantes en particular. Entre los determinantes implicados en el rendimiento académico, aparece el contexto socioeconómico y sociocultural de las familias, que justificarían la brecha educativa, sin olvidarnos de la participación de éstas en la escuela. En tal sentido, las abundantes investigaciones sobre la participación de los progenitores en el contexto escolar, nos conducen a una relación llena de encuentros y desencuentros. Pero cuando hablamos de las familias migrantes, dichos desencuentros se multiplican, caracterizándose en ocasiones por la frialdad y el desafecto, y con posturas poco dialogantes y constructivas. A menudo, se señala en este sentido, a las limitaciones y al uso inadecuado de las herramientas como la agenda, el teléfono o el correo electrónico, debido a las dificultades idiomáticas de las familias (Llevot y Bernand, 2015). 
En tiempos más recientes, se comenta que la repetición de contenidos y el carácter de mero trámite de las reuniones grupales, no ayuda a interiorizar nuevos conceptos ni a fomentar la participación (Bordalba, 2019; Chamseddine, 2020; Garreta, 2015). Algo que sustenta la falta de confianza y la ausencia del sentimiento de pertenencia de las familias al contexto escolar, que no incrementa el interés por estas vías de comunicación (Centorrino y Pellegrino, 2020; Molina, 2017). Por otra parte, autores como Santos-Rego, Lorenzo y Priegue (2009) sostienen que la baja formación de los progenitores condiciona su contribución en cuanto al grado de ayuda que pueden prestar a sus hijos e hijas en casa y más si se trata de contenido más complejos como en educación secundaria.

Otro punto crucial, apunta a la escasa participación de las familias migrantes en el Consejo Escolar, debido al desconocimiento del proceso a seguir para tener mayor presencia en el mismo, unido a que la información al respecto se transmite de forma deficiente cuando se trata de padres migrantes, ya que los docentes prefieren contar con las familias autóctonas en el Consejo Escolar (Andrés y Giró, 2016; Bolívar, 2006; Fernández, 2007; Martín y Gairín, 2007). En la misma línea, la representatividad de las familias migrantes en las AMPAs, es insuficiente donde predominan las familias autóctonas (Garreta, 2010; Gomáriz et al., 2008, 2019; Lozano, Alcaraz y Colás, 2013). Todo ello limita dicha relación a los contactos casuales en la entrada o salida del centro escolar, que son poco eficientes para abordar aspectos vinculados al grado de adaptación de los hijos, su desarrollo personal, sus capacidades o sus motivaciones.

Uno de los desafíos, sin duda, es revertir el discurso pesimista de los profesores y de las familias migrantes, que se ha instalado en el sentido fatalista. En tal sentido, los docentes se quejan de que los padres migrantes acuden al centro escolar, sólo en caso de incidencia o para abordar temas de calificaciones unido a la poca presencia de los varones en la dinámica escolar, y que frecuentemente las familias delegan en la escuela la misión de la educación de los hijos (González Falcón, 2007). Las familias migrantes, por su parte, manifiestan que la información que reciben de los docentes, es escasa y poco significativa, haciendo hincapié en la postura poco colaborativa del profesorado (Feito, 2007; Vallespir, Rincón y Morey, 2016), añadiendo que éste se centra más en el material escolar, dejando otros temas relevantes sin abordar (Essomba, 2014). A estas manifestaciones, se asocia que el profesorado no admite sugerencias que facilitan la fluidez de una participación activa, aferrándose al eterno discurso sobre la interferencia en su labor educativa (Bolívar, 2006). Además, a menudo los prejuicios y estereotipos de los docentes hacia las familias migrantes, se convierten en uno de los factores que más obstaculiza la comunicación y la contribución de dichos padres en el contexto escolar (González Facón, 2007). En tal línea de interés, Chamseddine (2018b) advierte que, si queremos que contribuyan a la mejora de dichos recursos, se debería profundizar en los intereses, necesidades y prioridades de las familias, así como en los propios contextos, y no limitarse únicamente a exteriorizar el fracaso y el abandono escolar en las familias o en su contexto social.

Como se ha comentado anteriormente, el sistema educativo adoptó una serie de acciones y medidas, como respuesta de la administración educativa para afrontar el aumento de alumnado migrante en las aulas. Como ejemplos tenemos el modelo de aulas temporales de adaptación lingüística de Andalucía, aulas de adaptación lingüística y social de CastillaLeón, aulas temporales de inmersión lingüística de Extremadura, aulas de enlace de Madrid, aulas temporales de inmersión lingüística de Asturias, aula de apoyo idiomático de Canarias, aula de acogida de la Región de Murcia, aulas de inmersión lingüística de Navarra y La Rioja, etc. Aunque tienen distintas denominaciones, pueden ser agrupadas 
todas ellas, bajo el concepto de aulas lingüísticas para el aprendizaje del castellano como lengua vehicular, como único cometido. Desde esta perspectiva, no facilitan la integración sociocultural del alumnado migrante como se pretendía en un principio, teniendo en cuenta que el apoyo se realiza fuera del aula ordinaria, siguiendo las directrices del enfoque segregacionista que genera aislamiento y desconexión (Nikleva, 2014), en lugar de implementar el modelo inclusivo, que se centra en la atención en el aula ordinaria, como la opción más idónea para facilitar la comunicación con los autóctonos. Algo que produce un impacto emocional significativo, en cuanto al proceso de construcción de identidad de los menores, inmerso en un escenario complejo que se canaliza hacia la identidad comunitaria, como única salida posible (Chamseddine, 2015, p. 201).

En un principio, la creación de dichas medidas pretendía favorecer el acceso, permanencia y promoción en el sistema educativo de dicho alumnado. No obstante, encontramos diferencias muy significativas entre las Comunidades Autónomas, donde el acceso atendiendo a la edad puede variar según cada Comunidad, ya que el programa sólo se aplica en Educación Secundaria, en algunas de ella, teniendo en cuenta que, en un principio, abarcaría también el segundo ciclo de educación primaria. También existen diferencias en el periodo de permanencia y la prórroga, donde unas permiten, un trimestre, otros dos trimestres e incluso tres trimestres. $\mathrm{Y}$ en cuanto a la promoción, existe una descoordinación palpable entre el profesor especialista y el del aula de referencia a la hora de incorporar el alumnado al grupo ordinario (Arroyo, 2011). Los profesores especialistas no disponen de un marco institucional adecuado para el desarrollo de funciones y tareas, que suelen ser desconocidas por los propios equipos directivos y los docentes de los centros educativos, lo cual dificulta las labores de coordinación y planificación de actuaciones (Guerrero, 2013). Esta ausencia de reconocimiento profesional, agudiza la presencia de conflictos y desmotivación de los propios docentes. Del mismo modo, aparecen diferencias de criterios azarosos entre Comunidades Autónomas, en cuanto al perfil y formación del profesorado. Se puede confirmar que el perfil de los docentes es diverso, y el requisito de la formación específica y la experiencia en educación compensatoria o en atención a la diversidad, no se exige ni se cumple en todas las Comunidades Autónomas, teniendo en cuenta las dificultades para afrontar la intervención educativa, la comunicación con las familias, y la coordinación con el resto de los docentes (Arroyo, 2011).

Asimismo, no dejan de ser paradójicos los criterios establecidos para solicitar la aplicación de dichos programas. En cada Comunidad Autónoma se exige un número determinado de alumnos, que pueden ser 6, 10, 15, 20 o 30, con un informe psicopedagógico previo, y de conformidad con la familia, etc., que a menudo no se adecua a la realidad de dichos centros, ni se dotan de los recursos suficientes. En ocasiones el docente es compartido con otros dos institutos, donde apenas superan las diez horas semanales, cuando la asistencia a estos grupos es de 10 horas semanales en educación primaria y 15 en Educación Secundaria. En síntesis, digamos que pocas son las voces que se atreven a defender estos programas y medidas de mayor discusión, controversia sin evaluación, ni seguimiento exhaustivo. Arroyo (2011), advierte sobre la ausencia de reflexión crítica acerca de la viabilidad de dichas medidas, en cuanto a aspectos vinculados a la inclusión educativa, basada en los cambios adecuados, con programas curriculares docentes, con objetivos claros, reales y alcanzables. 
Otro factor estructural, que viene a reforzar los determinantes señalados anteriormente es la escasa formación inicial, como permanente del profesorado, cuanto en la atención a la diversidad cultural en las aulas, que a menudo estimula la frustración y poca implicación de estos profesionales que se ven desbordados por la situación (Santos y Lorenzo, 2009). Desde luego, un examen exhaustivo de los planes de formación de las distintas universidades españolas, nos remiten a que la asignatura de educación intercultural aparece como materia optativa o de libre configuración. Lo que importa es formar a profesionales para afrontar las nuevas realidades en los centros educativos, y que sean capaces de generar diálogos críticos, y resilientes entre alumnados procedentes de diversas culturas (Massot et al., 2013; Molina-Pérez y Luengo, 2020). Formación cuyo pilar es transmitir conocimientos, habilidades y actitudes que conducen a la construcción un docente inclusivo capaz de articular la relación entre formación inicial y desarrollo profesional con la idea esencial de la transformación educativa a través de prácticas pedagógicas de los siguientes propósitos (Echeita, 2012):

\section{Propósitos afectivos, reforzando la seguridad, la confianza, el sentido de competencia y de control y el interés; propósitos sociales, incrementando el sentido de aceptación, reconocimiento, valoración, y pertenencia a una comunidad/grupo; y propósitos intelectuales, asegurando mejorar el acceso, el significado, la aplicación y el razonamiento relevante. (p. 15)}

Otro de los problemas estructurales no resueltos, es la necesidad de profundizar en los efectos de la discontinuidad del profesorado con experiencia en los centros educativos, ubicados en contextos vulnerables, y las consecuencias de sustituirles por profesores nóveles -algo que hemos detectado en numerosos centros educativos de la Región de Murcia, y que apenas existen investigaciones empíricas al respecto-. La gestión del profesorado interino, en ocasiones insuficiente e inadecuada, genera un cambio de alumnos, de centro, de compañeros y de contexto escolar, que se da incluso varias veces en el mismo curso escolar, donde con frecuencia, el docente interino debe ceñirse a una programación ajena, y por criterios pedagógicos del titular de la plaza. Prescindir de este profesorado con experiencia en centros con mayor concentración de alumnado migrante, para sustituirles por un profesorado novel, que tiene que adaptarse a una serie de demandas y exigencias de los centros educativos en contextos desfavorecidos, repercute en nuestra opinión, negativamente en todos los actores implicados en el proceso formativo de los alumnos migrantes. Del mismo modo, creemos que aumenta la probabilidad de fracaso escolar de los mismos. Es preciso, por tanto, garantizar que dichos centros cuenten con la selección de los mejores profesionales, así como promover la estabilidad laboral de los mismos.

Unido a estos hechos consideramos preciso referir la escasa e intermitente presencia de la figura del mediador o mediadora intercultural en los centros educativos. Se trata de una profesión emergente en proceso de construcción (Iglesias, Pastor y Rondón, 2017). Su cometido como soporte de apoyo especializado es esencial para los equipos directivos y docentes (Martínez, Sahuquillo y García, 2012), así como para las propias familias migrantes (Casasempere, 2013). Su liderazgo inclusivo (Fernández Enguita, 2007; Rodorigo, Fernández-Larragueta y Fernández-Sierra, 2019), y transformador de la realidad social (Fernández-Larragueta, Rodrigo y Fernández Sierra, 2014), permite clarificar peculiaridades culturales y eliminar las barreras lingüísticas (Catarci, 2016), para impulsar los cauces participativos que consoliden la convivencia intercultural en los contextos escolares (Ibarrola e Iriarte, 2013; Ospina, 2016). En tal sentido, Chamseddine (2018a) matiza que la función del mediador o mediadora intercultural: 
No es una mera traducción simultánea o escrita, ni es sólo un instrumento de resolución de conflictos de carácter cultural, sino un proceso que promueve estrategias de participación, intercambio e interacción cuyo propósito es el conocimiento, el reconocimiento y la valoración de la diversidad cultural desde la igualdad de oportunidades para lograr la convivencia, la cohesión y la transformación social productiva. (p. 200)

Sin embargo, el carácter intermitente y precario a nivel laboral de la figura del mediador o mediadora intercultural, está asociado a una contratación puntual, articulada a través de programas y subvenciones impulsados con fondos de la administración pública. El resultado de este escenario obstaculiza las peticiones y demandas de los centros educativos, que precisamente no son periódicas (Llevot y Garreta, 2013). A diferencia de otros contextos europeos, donde esta figura profesional se encuentra institucionalizada dentro del sistema escolar.

\section{Para concluir}

Sabemos que, hoy no falta quien presente la migración como amenaza con una lectura pesimista e institucionalizada, convertida en una mera arma electoral donde se emplea la diferencia cultural, para establecer la marginación y la exclusión social. Por tanto, sería acuciante reconvertir esta tendencia creciente que aborda la migración desde la criminalización y la seguridad nacional, generando tensiones y miedos, a una gobernanza de la migración, que priorice sus derechos e intereses, haciendo hincapié en su aportación a la estructura económica y social (Canales, 2013).

De tal posicionamiento excluyente, no se libran ni los niños y niñas de origen migrante ni sus familiares, algo que se ve reflejado en el discurso tozudo e irreconciliable de los centros educativos en general, y del propio profesorado en particular, sustentados por la desentonación temeraria producida en las últimas tres décadas en la gestión educativa y administrativa de dicha materia. Ese empeño de implantar y legitimar las desigualdades en la dinámica escolar, modifica el recorrido de unos estudiantes que pasan, teóricamente, hablando, de situaciones de inclusión educativa a situaciones de exclusión escolar, y por tanto social. En concreto, y al volver la mirada hacia lo expuesto, es ineludible repensar y replantear el modelo de inclusión escolar que se requiere. Y para ello, no hay que olvidar la cooperación en la práctica cotidiana entre los distintos agentes educativos y sociales, especialmente, en la gestión eficiente de las políticas educativas que se establecen en materia de alumnado de origen migrante (Fernández, 2018).

Por todo ello, lo cabal sería garantizar una escuela accesible, en igualdad de condiciones y más aún a los grupos más vulnerables, adaptable a los intereses, necesidades y prioridades de los alumnos, de las familias migrantes y del propio contexto social de los centros educativos, y alternativa, con un profesorado bien preparado a nivel formativo y estable laboralmente. Una escuela que apuesta por la innovación y la investigación en nuevos métodos de enseñanza-aprendizaje y capaz a su vez, de dedicar un mayor esfuerzo a conocer a cada alumno y su estilo de aprendizaje, habilidades, potencialidades y limites (Chamseddine, 2018b; Chamseddine y Hernández, 2020). En la misma línea, Massot y otros (2013), nos ofrecen las siguientes propuestas de actuación a implementar:

I. Fortalecer las condiciones individuales que pueden promover la resiliencia y asegurar un éxito en la transición académica y profesional.

II. Potenciar el desarrollo de un sentimiento de pertenencia. 
III. Estimular la participación en las dinámicas del centro.

$I V$. Potenciar los vínculos escuela-comunidad mediante los planes de entorno o las actividades de aprendizaje-servicio.

$V$. Promover procesos óptimos de orientación y transición académica y profesional.

VI. Potenciar la incorporación de las familias en los procesos orientadores del alumnado.

VII. Diseñar e implementar estrategias, actividades y programas que den la oportunidad de responder con éxito al estudiante.

VIII. Favorecer espacios y trayectorias que retornen de forma realista y sostenida una imagen de posibilidad y autoeficacia personal y académica.

IX. Implementar programas de formación y/o asesoramiento en resiliencia, tanto para los profesionales en ejercicio como en formación inicial. (pp. 73-74)

Expuesto lo anterior, no queremos terminar sin decir que toda previsión de futuro no deja de resultar un ejercicio arriesgado que, en este caso nos preocupa, y que está sujeto a una mayor incertidumbre, debido a los múltiples determinantes que se deberían afrontar. Además, cabe recordar, que los desafíos demográficos imponen la baja tasa de natalidad y el progresivo envejecimiento de la población. Partiendo de esta premisa, sería poco inteligente despreocuparnos del rendimiento y del éxito académico de dicho alumnado, por ende, en las graves consecuencias que puede generar el cultivo de futuros ciudadanos, -por supuesto españoles- no cualificados que les será muy complejo insertarse en el mundo laboral, dada la demanda existente cada vez más exigente y competitiva.

Permítasenos insistir en desalojar las malas prácticas organizativas, estructurales y curriculares, que estimulan el fracaso escolar de determinados grupos culturales, y que evidentemente forma parte de su fracaso social, contribuyendo a producir toda una generación de personas migrantes severamente castigada, por un elevado riesgo de instalarse en empleos precarios, y sin expectativas de promoción profesional. Por ello, construir desde la equidad la inclusión de estas minorías culturales o étnicas debería convertirse en un imperativo ético y moral para una escuela más justa, y democrática para evitar por acción o por omisión la reproducción de las injusticias sociales (Murillo y Hernández, 2014). Por tanto, lo sensato y perentorio sería visibilizar y garantizar la promoción integral de la diversidad cultural dentro y fuera del aula, propiciando la inclusión educativa y equitativa, y minimizando las condiciones que posibiliten la exclusión escolar y la reproducción de la desintegración de la segunda generación que son los españoles del mañana.

En síntesis, existe una necesidad palpable por el "despertar" del interés por el liderazgo en los centros educativos con un propósito moral para la justicia social, especialmente en contextos de diversidad brechas socioeconómicas, cultivar la inclusión, reflexión crítica de estilos, actitudes y comportamientos para conseguir la transformación de situaciones injustas (González González, 2014). En la misma línea argumental, Queupil y Montecinos (2020) matizan sobre la necesidad de generar condiciones para la distribución de una red de liderazgo entre los equipos directivos y departamento cohesionados respecto a cómo se puede abordar esas "nuevas" realidades educativas. Es imperativo, por tanto, potenciar un lenguaje común en la práctica educativa, concebir y sensibilizar que la diferencia es una oportunidad para el aprendizaje, y fortalecer una cultura escolar compartida para una sociedad inclusiva a la que no debemos renunciar. 
La limitación de la presente aportación estriba en la ausencia de datos desagregados y viables en las estadísticas consultadas del Ministerio de Educación en relación al alumnado extranjero. Asimismo, sería de interés llevar a cabo, entrevistas semiestructuradas o grupos de discusión a informantes claves de la comunidad educativa, para conocer en mayor profundidad las dinámicas que explican de forma exhaustiva la segregación y exclusión escolar de este alumnado, y que indudablemente enriquecería la síntesis teórica realizada.

Para futuras investigaciones en dicha materia, sería importante analizar con mayor rigurosidad variables como la procedencia del alumnado extranjero, el liderazgo de los equipos directivos para impulsar la implicación de las familias extranjeras en la vida del centro escolar, el perfil formativo de los docentes, la discontinuidad del profesorado con experiencia profesional en contextos vulnerables, la institucionalización de la figura del mediador o mediadora intercultural, y la influencia de las distintas modalidades de aulas lingüísticas en la inclusión o exclusión de este alumnado.

\section{Referencias}

Alegre, M. A (2010). Casi-mercados, segregación escolar y desigualdad educativa: Una trilogía con final abierto. Revista Educación y Sociedad, 113(31), 1157-1178. https://doi.org/10.1590/So101-73302010000400006

Andrés, S. y Giró, J. (2016). El papel y la representación del profesorado en la participación de las familias en la escuela. Revista Electrónica Interuniversitaria de Formación del Profesorado, 19(1), 61-71. https://doi.org/10.6018/reifop.19.1.245461

Arroyo, M. J. (2011). Las aulas y programas de inmersión lingüística para alumnado extranjero en España. Revista Segundas Lenguas e Inmigración en Red, 5, 114-139.

Bolívar, A. (2006). Familia y escuela: Dos mundos a trabajar en común. Revista de Educación, 339, 119-146.

Bordalba, M. (2019). Principales canales para la comunicación familia-escuela: Análisis de necesidades y propuestas de mejora. Revista Complutense de Educación, 30(1), 147-165. https://doi.org/10.5209/RCED.56034

Canales, A. (2013). Migración y desarrollo en las sociedades avanzadas. Revista Polis, 35, art. 5. https://doi.org/10.4067/S0718-65682013000200005

Casasempere, A. V. (2013). La mediación intercultural como agente articulador de las relaciones positivas entre la familia de origen migrante y los centros educativos. En M. C. Cardona E. Chiner y A. Giner (Coords.), Actas del XVI Congreso Nacional y II Internacional Modelos de Investigación Educativa de la Asociación Interuniversitaria de Investigación Pedagógica (AIDIPE) (pp. 88-94). Universidad de Alicante.

Catarci, M. (2016). Intercultural mediation as a strategy to facilitate relations between the school and immigrant families. Revista Electrónica Interuniversitaria de Formación del Profesorado, $19(1), 127-140$.

Centorrino, M. y Pellegrino, L. (2020). Scuola e famiglie immigrate: Uno studio in una scuola del sud Italia escuelas y familias inmigrantes. Revista Educatio Siglo XXI, 38(1), 55-82. https://doi.org/10.6018/educatio.403551

Chamseddine, M. (2015). La construcción de identidad compartida en un aula intercultural. Revista Electrónica Interuniversitaria de Formación del Profesorado, 18(3), 69-81.

https://doi.org/10.6018/reifop.18.3.238841 
Chamseddine, M. (2018a). Polarización escolar en España. Retos e implicaciones. International Journal of Educational Research and Innovation, 9, 189-205.

Chamseddine, M. (2018b). Principios inherentes a la mediación intercultural en la escuela. Revista Mediaciones Sociales, 17, 1-19. https://doi.org/10.5209/MESO.58512

Chamseddine, M. (2020). Study on communication between migrant families and schools. Revista Electrónica Interuniversitaria de Formación del Profesorado, 23(1), 34-47. https://doi.org/10.6018/reifop.404181

Chamseddine, M. y Hernández, A. (2020). Actitudes de los escolares ante la llegada de población migrante: Estudio comparativo y aproximación al desarrollo de buenas prácticas educativas. Revista Educatio Siglo XXI, 38(1), 229-252. https://doi.org/10.6018/educatio.413491

Commission des Communautés Européennes. (2008). Livre vert. Migration et mobilité: Enjeux et opportunités pour les systèmes éducatifs européens. COM.

Consejo Económico y Social de España. (2019). Informe de la inmigración en España: Efectos y oportunidades. NICES.

Cucalón Tirado, P. y Del Olmo, M. (2019). Dilemas y tensiones en la práctica docente con alumnado migrante. Revista Disparidades, 74(1), 1-19.

https://doi.org/10.3989/dra.2019.01.007

Escudero, J. M (2006). Compartir propósitos y responsabilidades para una mejora democrática de la educación. Revista de Educación, 339, 19-41. https://doi.org/10.4995/redu.2014.5636

Escudero, J. M. (2014). Calidad y equidad en la enseñanza universitaria. REDU. Revista de Docencia Universitaria, 12(2), 11-13.

Essomba, M. A. (2014). Políticas de escolarización del alumnado de origen extranjero en el estado español hoy. Análisis y propuestas. Revista Electrónica Interuniversitaria de Formación del Profesorado, 17(2), 13-27. https://doi.org/10.6018/reifop.17.2.198771

Feito, R. (2007). Balance de la participación de los padres en los consejos escolares de centro. Participación Educativa, 4, 4-15.

Fernández Enguita, M. (2007). Educar es cosa de todos: Escuela, familia y comunidad. En J. Garreta (Ed.), La relación familia escuela (pp. 57-78). Edicions de la Universitat de Lleida.

Fernández-Larragueta, S., Rodrigo, M. y Fernández Sierra, J. (2014). La mediación intercultural en la escuela: Una práctica en construcción. Revista En-clave Pedagogica, 13, 67-75.

Fernández, M. (2018). Bases para acordar el futuro de la educación. Cuadernos de Pedagogía, 488, 74-78.

Franzé, A., Poveda, D., Jociles, M. I., Rivas, A. M., Villaamil, F., Peláez, C. y Sánchez, P. (2012). La segregación étnica en la educación secundaria de la ciudad de Madrid: Un mapa y una lectura crítica. En F. J. García Castaño y S. Carrasco Pons (Eds.), Población inmigrante y escuela: Conocimientos y saberes de investigación (pp. 119-134). IFIIE-CREADE.

García, F. J., Olmos, A., Rubio, M. y Capellán, L. (2014). Sobre agrupamiento, concentración, segregación o guetización escolar: Claves para un análisis interpretativo de tales situaciones y procesos. En C. Blanco-Fernández (Coord.), Movilidad humana y diversidad social en un contexto de crisis económica internacional (pp. 253-276). Trotta.

Garreta, J. (2010). La participación de las familias en la escuela. En R. Feito (Coord.), Sociología de la educación secundaria (pp. 47-65). Graó.

Garreta, J. (2015). La comunicación familia-escuela en educación infantil y primaria. RASE. Revista de Sociología de la Educación, 8(1), 71-85. 
Gomariz Vicente, M. A., Martínez-Segura, M. J. y Parra Martínez, J. (2019). Desde la implicación en el hogar de las familias a la facilitación de los docentes en un contexto multicultural. Revista Electrónica Interuniversitaria de Formación del Profesorado, 22(3), 45-60. https://doi.org/10.6018/reifop.389631

Gomariz Vicente, M. A., Parra Martínez, J., García Sanz, M. P., Hernández Prados, M. A. y Pérez Cobacho, J. (2008). La comunicación entre la familia y el centro educativo. Consejo Escolar de la Región de Murcia.

González Facón, I. (2007). La participación de las familias inmigrantes en la escuela: Necesidades de orientación y formación. XXI. Revista de Educación, 9, 155-169.

González González, M. T. (2014). El liderazgo para la justicia social en organizaciones educativas. Revista Internacional de Educación para la Justicia Social, 3(2), 85-106.

Guerrero Valdebenito, R. M. (2013). Integración sociocultural y enseñanza del español en Andalucía: aplicación del programa aulas temporales de adaptación lingüística (ATAL). Revista Perfiles Educativos, 35(142), 42-53. https://doi.org/10.22201/iisue.24486167e.2013.142.42574

Ibarrola, S. y Iriarte, C. (2013). La influencia positiva de la mediación escolar en la mejora de la calidad docente e institucional: Percepciones del profesor mediador. Revista Profesorado. Curriculum y Formación del Profesorado, 17(1), 367-381.

Iglesias, E., Pastor, E. y Rondón, L. M. (2017). Mediación como profesión emergente: Actualidad formativa desde la educación superior. Revista Mediaciones Sociales, 16, 135-153. https://doi.org/10.5209/MESO.58113

Llevot, N. y Bernad, O. (2015). La participación de las familias en la escuela: Factores claves. RASE. Revista de Sociología de la Educación, 8(1), 57-70. https://doi.org/10.3989/ris.2012.09.07

Llevot, N. y Garreta, J. (2013). La mediación intercultural en las asociaciones de inmigrantes de origen africano. Revista Internacional de Sociología, 71(1), 167-188. https://doi.org/10.3989/ris.2012.09.07

Lozano Martínez, J., Alcaraz García, S. y Colás Bravo, M. P. (2013). Los centros educativos multiculturales y sus relaciones con las familias: El caso de la región de Murcia. Revista Educación XX1, 16(1), 210-232. https://doi.org/10.5944/educxx 1.16.1.724

Machado, C. (2015). Inmigración y universidad. Una aproximación al caso de la Universidad de La Laguna. Boletín Millares Carlo, 31, 252-285. https://doi.org/10.17533/udea.boan.v31n52a18

Martín, E. (2010). Actores y mecanismos de categorización de los hijos de familias inmigrante en la escuela. Revista del Ministerio de Trabajo e Inmigración, 90, 41-58.

Martín, M. y Gairín, J. (2007). La participación de las familias en la educación: Un tema por resolver. Revista Bordón, 59(1), 113-151.

Martínez, M. J., Sahuquillo, P. y García, L. (2012). Identidad y responsabilidades socioeducativas del mediador escolar y del mediador intercultural: Hacia una clarificación de funciones. Revista Mediaciones Sociales, 11, 47-71. https://doi.org/10.5209/rev_MESO.2012.v11.41269

Massot, M. I., Del Campo, J., Sandín, M. P y Sánchez, A. (2013). Resiliencia y éxito escolar en el alumnado de secundaria de procedencia extranjera. En M. Cardona, E. Chiner y A. Giner (Coords.), Actas del XVI Congreso Nacional y II Internacional Modelos de Investigación Educativa de la Asociación Interuniversitaria de Investigación Pedagógica (AIDIPE) (pp. 67-75). Universidad de Alicante. 
Molina, J. (2017). Los organismos independientes en la democracia representativa. Revista de Investigación en Tecnologías de la Información, 3, 1-10.

Molina-Pérez, J, y Luengo. (2020). Reconstrucciones resilientes de la identidad profesional del profesorado: Endoprivatización y cultura performativa en Andalucía (España). REICE. Revista Iberoamericana sobre Calidad, Eficacia y Cambio en Educación, 18(2), 57-75. https://doi.org/10.15366/reice2020.18.2.003

Moreno, M. A. (2002). Neoliberalismo, escuela pública y educación intercultural: Contexto e implicaciones. Universidad de Murcia.

Murillo, F. J. y Hernández, R. (2014). Liderando escuelas justas para la justicia social. Revista Internacional de Educación para la Justicia Social, 3(2), 13-32.

Murillo, F. J., Martínez-Garrido, C. y Belavi, G. (2017). Segregación escolar por origen nacional en España. OBETS: Revista de Ciencias Sociales, 12(2), 395-423.

https://doi.org/10.14198/OBETS2017.12.2.04

Nikleva, D. G. (2014). El reto de atención a alumnos inmigrantes en la sociedad española. Editorial Síntesis

Ortiz, M. (2010). Ineficacia de las medidas para garantizar la igualdad de oportunidades. Revista Iberoamericana de Educación, 51(3), 1-7. https://doi.org/10.35362/rie5131829

Ortiz, M. (2014). Inmigración, escuela y exclusión. Revista Empírea de Metodología de Ciencias Sociales, 28, 59-78. https://doi.org/10.5944/empiria.28.2014.12121

Ospina, Y. (2016). Tensiones de los sujetos...en la convivencia escolar. Revista de Educación y Pensamiento, 23, 79-91.

Queupil, J. y Montecinos, P. (2020). El liderazgo distribuido para la mejora educativa: Análisis de redes sociales en departamentos de escuelas secundarias chilenas. REICE. Revista Iberoamericana sobre Calidad, Eficacia y Cambio en Educación, 18(2), 97-114. https://doi.org/10.15366/reice2020.18.2.005

Rodorigo, M., Fernández-Larragueta, S. y Fernández-Sierra, J. (2019). La mediación intercultural como herramienta de inclusión: Análisis de una experiencia escolar. Revista Complutense de Educación, 30(3), 747-761. https://doi.org/10.5209/rced.58885

Rubia, F. A (2013). La segregación escolar en nuestro sistema educativo. Fórum Aragón, 10, 47-52.

Salinas Jiménez, J. y Santín González, D. (2012). Selección escolar y efectos de la inmigración sobre los resultados académicos españoles en PISA 2006. Revista de Educación, 358, 382-405.

Sánchez Santamaría, J. y Manzanares, A. (2012). La equidad educativa: Dilemas, controversias e implicaciones para garantizar el éxito educativo para todos. En A. Manzanares (Coord.), Temas educativos en el punto de mira (pp. 49-75). Wolters Kluwer.

Santos Rego, M. A. y Lorenzo Modelo, M. M. (2009). La participación de las familias inmigrantes en la escuela: Un estudio centrado en la procedencia. Revista de Educación, 350, 277-300.

Vallespir, J., Rincón, J. C. y Morey, M. (2016). La participación de las familias en el consejo escolar y la formación del profesorado. Revista Electrónica Interuniversitaria de Formación del Profesorado, 19(1), 31-45. https://doi.org/10.6018/ reifop.19.1.245751

Vecina, C. (2005). Jóvenes inmigrantes en la Universidad de Las Islas Baleares. El privilegio de la distinción. Revista Aposta de Ciencias Sociales, 16, 1-21. 


\section{Breve CV del autor}

\section{Mohamed Chamseddine}

Doctor en Educación, Licenciado en Pedagogía, Diplomado en Educación Social, y Máster en Intervención Social y Mediación. Especialista Universitario Consultor sobre Migraciones y Acción Solidaria. Profesor en el Departamento de Didáctica y Organización Escolar en la Facultad de Educación de la Universidad de Murcia. Sus investigaciones están vinculadas a la Educación Intercultural, Mediación Intercultural y Educación Inclusiva de Colectivos Vulnerables. Miembro del Grupo de Investigación Educación Inclusiva: Escuela para Todos (Grupo EDUIN, E073-02), y miembro de la Asociación de Expertos y Evaluadores de la Unión Europea (EVALUE). ORCID.ID: https://orcid.org/0000-0001-9817-652X. Email: mohamed.c.h@um.es 